\title{
Conservation of the role of INNER NO OUTER in development of unitegmic ovules of the Solanaceae despite a divergence in protein function
}

\author{
Debra J. Skinner ${ }^{1}$, Ryan H. Brown ${ }^{1,2}$, Robert K. Kuzoff',3 and Charles S. Gasser ${ }^{1 *}$
}

\begin{abstract}
Background: The INNER NO OUTER (INO) gene is expressed in the outermost cell layer of the outer integument of bitegmic ovules and is essential for this organ's growth. The role and cross-species functional conservation of INO orthologs were examined in members of the Solanaceae, which have unitegmic ovules. Unitegmy has evolved several times in disparate angiosperm lineages. INO expression has been observed in the outermost cell layers of all examined unitegmic ovules, but the functional role of INO in unitegmic ovules has not previously been evaluated.

Results: INO orthologs were unambiguously identified in tobacco and tomato by sequence homology. Expression of the tomato INO gene was limited to the outer cell layer of the single integument indicating that this single integument has properties of the outer integument. Expression occurred only after integument initiation, later than observed in ovules of other examined angiosperms. Virus-induced knock-down of expression of the INO ortholog in tobacco inhibited growth of the outer cell layer of the integument leading to a decrease in both integument extension and curvature of the ovule. The altered ovules closely resemble those of the aberrant testa shape (ats) ino mutant combination in Arabidopsis where we see the effect of the ino mutation on a single fused integument produced by the ats mutation. Despite significant sequence identity and similar expression patterns, the tomato INO coding region was not able to complement the Arabidopsis ino mutant.

Conclusions: The similarity of effects of ino mutations on the unitegmic ovules of tobacco and the fused integuments of the Arabidopsis ats mutant show that: 1) INO orthologs play the same role in promoting integument growth in ovules of tobacco and Arabidopsis; and 2) the unitegmic ovules of tobacco (and hence other solanaceous species) are most likely the result of a congenital fusion of two ancestral integuments. Our results further indicate that INO has a conserved role in growth of the outermost cell layer of integuments. The curvature of solanaceous ovules is driven by unequal growth of the outer layers of the single integument that likely correspond to an ancestral outer integument.
\end{abstract}

\section{Background}

Among seed plants, ovule structure is largely conserved in comprising three main structures: the nucellus, site of megagametophyte development; the integument(s), sheath(s) of cells that surround the nucellus; and the funiculus, a stalk providing a physical and vascular connection to the placenta. In the angiosperms, significant variation in ovule form occurs in the number

\footnotetext{
* Correspondence: csgasser@ucdavis.edu

${ }^{1}$ Department of Molecular and Cellular Biology, University of California, Davis, Davis, CA 95616, USA

Full list of author information is available at the end of the article
}

and thickness of integuments and curvature of the ovule [1]. The earliest diverging angiosperm lineages are largely bitegmic [2, 3] and anatropous (curved toward the placenta), two characters that are likely to be intimately connected [4]. Ovule curvature is often caused by asymmetric integument growth, usually of the outer integument as seen, for example, in bitegmic ovules of Arabidopsis. The outer integument initiates in a semi-annular pattern around the chalaza and continues to grow only on the gynobasal (toward the base of the gynoecium) side of the ovule. In Arabidopsis outer integument initiation and growth require the activity of 
the INNER NO OUTER (INO) gene, expressed at the site of integument initiation and persisting in the outer layer of the growing outer integument [5, 6]. Arabidopsis mutants lacking a functional copy of INO fail to produce an outer integument. Additionally, in the superman mutant symmetric expression of AtINO around the chalaza leads to development of symmetric outer integument, and symmetric largely orthotropous ovules.

INO expression has been studied in a range of taxa, including early diverging bitegmic angiosperms such as Cabomba caroliniana (CcINO) (Nymphaeales) and Annona squamosa (Magnoliales) and in these early lineages, the expression of INO in the outermost cell layer of the outer integument is conserved $[7,8]$. Moreover, a unitegmic $A$. squamosa mutant lacks the INO gene and the outer integument is absent, suggesting that the role of $I N O$ in outer integument growth was established early in the angiosperm lineage [7]. Thus, INO expression can be used as a practical marker for the outer integument that is useful in understanding the nature and evolution of integuments in angiosperms.

Where such genes show conservation of expression and function across diverse taxa, it is informative to ask how that function has been maintained at the level of regulation of expression and the activity of the protein. Cross species complementation can indicate whether proteins have diverged in their targets and activity. Similar experiments and examination of alignments between promoters can indicate regions that retain a shared regulatory function.

Unitegmy has arisen from the ancestral bitegmic state in several angiosperm lineages through different proposed mechanisms such as fusion of the two integuments, loss of an integument, or integumentary shifting [9]. Unitegmy is a shared character of the euasterids, indicating a single origin of this character for this clade [10], but the derivation of this morphology in euasterids has not yet been elucidated. The closest relatives of the euasterids that retain bitegmic ovules are in the Ericales grade, the basal lineages in the broader asterid clade, where several genera have bitegmic ovules [10]. We previously examined species with unitegmic, bitegmic and intermediate ovules in Impatiens, a genus within the Ericales [11]. We found that integument fusion was the cause of derived unitegmy in this genus, and that anatropous curvature of all of these ovules derived primarily from curvature of the present, or ancestral outer integument [11].

Ovule development is nearly invariant in the euasterids, even in members of the most distantly related clades $[10,12]$, such as Aster and Solidago in the Asterales [13], Antirrhinum [14] in the Lamiales, and Solanum lycopersicum (tomato) $[15,16]$ in the Solanales. Ovule development among members of the Solanales such as as tomato $[15,16]$, tobacco [17] and petunia $[18,19]$, is sufficiently invariant that a description of one species can suffice for all three. In tomato, rows of ovule primordia arise on the central placenta during floral stage 8 , and the megaspore mother cell differentiates, with one layer of nucellar cells covering the megaspore $[15,16]$. As the megaspore becomes visible, the ovule primordium grows asymmetrically, leading to a slightly curved primordium. The integument primordium arises just below the level of the megaspore during stage 11 on the thickened side of the ovule. The integument grows asymmetrically to cover the nucellus and at maturity the ovule is fully curved with the micropyle adjacent to the placenta. The funiculus remains short throughout ovule development. Tomato is a good research model for euasterids due to the recent sequencing of the tomato genome [20] and the existence of many phenotypic variants (TGRC.ucdavis.edu), while tobacco has established methods for gene function evaluation through Virus Induced Gene Silencing (VIGS, [21]). In this study we examined the role of the INO gene in the unitegmic solanaceous species tomato and tobacco during integument development by assessing gene expression, function and sequence conservation.

\section{Results}

\section{Isolation of solanaceous INO sequences}

The tomato (Solanum lycopersicum) genome includes nine YABBY genes [20]. In a phylogenetic analysis of these genes, together with representative genes from other angiosperms, five clades were identified [22, 23], (Additional file 1: Figure S1), consistent with prior analyses of the gene family in other angiosperms $[8,24]$. While there was only a single tomato gene in the INO clade (SIINO), the other sub-families of YABBY genes: CRC, YAB2, YAB3/FIL, and $Y A B 5$ were each represented by two genes in tomato (Additional file 2: Table S1). To facilitate characterization, SIINO cDNA was isolated from a tomato pistil cDNA library [25] by hybridization with the Arabidopsis INO cDNA. This coding sequence confirmed the sequence of the predicted protein identified as SlINO (Solyc05g005240) in the tomato genome (Solgenomics.net, [22]). Using SIINO cDNA as a probe, a genomic clone of SIINO that included the first four exons and the 5'flanking region up to the adjacent predicted gene was isolated from a genomic library [26].

Nicotiana benthamiana is a recent allotetrapoid and includes two paralogous copies of each of the YABBY genes (Solgenomics.net, [27]). Consistent with this, two INO homologs were identified by homology searches of the draft sequence and predicted genes at solgenomics.net: Niben101Scf09599g00012.1 (NbINO1) and Niben101Sc f04287g04009.1 (NbINO2). NbINO1 appears to be incorrectly annotated, based on alignment with SIINO and AtINO, and is more likely to be as shown in Additional file 3: Figure S2. The two encoded INO proteins are $95 \%$ 
identical to each other and are $76 \%$ identical and $84 \%$ similar to SIINO along their aligned length, and clearly group with other INO proteins in phylogenetic analysis (Additional file 1: Figure S1). Polymerase chain reaction was used to isolate a partial genomic clone of NbINO1 for use in VIGS.

\section{Conservation of INO expression in tomato}

A feature of characterized INO genes is specific expression in the outer layer of the outer integument or single integument of ovules, with this pattern being conserved among eudicots like Arabidopsis [6], Impatiens [11] and Prunus [28] and members of early branching groups such as the Magnoliales (Annona [7]) and Nymphaeales (Cabomba [8]). Expression profiling showed expression of the S. pimpinellifolium (wild tomato LA1589) INO ortholog to be limited to flowers during the period of ovule development and two days post anthesis, with the peak of expression at anthesis [22, 29]. In situ hybridizations using the SIINO cDNA were performed on tomato carpels (Fig. 1a-c). SlINO transcripts were only detected in ovules, where they were confined to the outermost layer of the single integument, and were not observed in early ovule primordia. These results suggest that in the unitegmic tomato, the integument has characteristics of the outer integument, similar to results seen in unitegmic Impatiens and Prunus species [11, 28].

For further expression analysis and for heterologous expression, a putative promoter region including $2257 \mathrm{bp}$ of 5 ' flanking and untranslated sequence (P-SIINO) extending to the next upstream gene was isolated from the genomic clone. This region was used to drive transcription of green fluorescent protein (GFP) fusion genes in tomato (Fig. 2). P-SIINO::SlINO:GFP gave results similar to the in situ hybridization. Expression was detected only in the outer cell layer of the integument and was first visible after the emergence of the integument (Fig. 2b, c). Expression was low or absent from the few cells at the distal tip of the integument (Fig. 2 all stages), and also absent from integument cells on the side of the ovule opposite from the side on which most growth occurs (Fig. 2j, k). Expression in the outer layer of the integument remained high in mature ovules at anthesis (Fig. 2k) and continued to be visible through the onset of fruit development (Additional file 4: Figure S3). These results showed that the 5'-flanking and 5'-untranslated regions of SIINO contained sufficient information to duplicate the in situ results. The expression pattern is similar to that seen in Arabidopsis [6], Impatiens [11], Prunus [28], Annona [7] and Cabomba [8], differing from Arabidopsis in that SIINO expression was not detected prior to integument initiation, was maintained for longer during development, and the signal was spread over nearly the entire surface of the integument, rather than being concentrated toward the base of the integument.

\section{Conservation of role of INO in ovule development}

The similarities between the expression pattern and protein sequence of the tomato and Arabidopsis $I N O$ genes indicated that SIINO might play a role in tomato integument growth. Such a role would likely be conserved in closely related unitegmic solanaceous plants. As mutants of $I N O$ were not available in such species, virus induced gene silencing (VIGS) was used to engineer a knock-down of INO function in $N$. benthamiana. VIGS is especially effective in this species [30], including for the study of development [31]. N. benthamiana ovules [17] are very similar to those of tomato ([15, 16], and compare Fig. $2 \mathrm{f}, \mathrm{j}$,
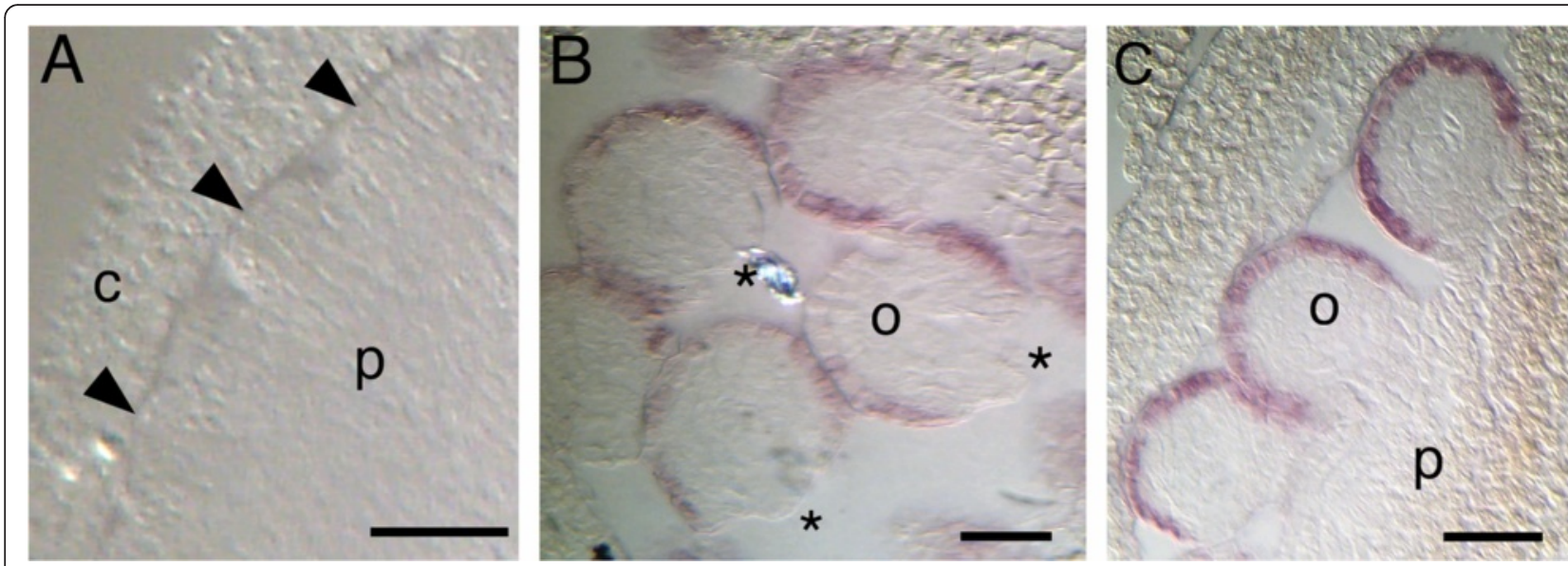

Fig. 1 Integument specific expression of SIINO. a-c In-situ hybridization with a labeled SIINO antisense RNA probe in tomato ovules. Purple stain indicates hybridization to SIINO mRNA. a Longitudinal section of stage 8 carpel with ovule primordia (arrowheads) prior to integument initiation showing no hybridization signal. b Cross-section of stage 14 ovules with exposed nucelli ${ }^{*}$ ) showing hybridization signal in outermost cell layer of the integument. c Stage 16 ovules show the specificity of the SIINO expression pattern, with no hybridization in placenta, funiculus or inner cell layers of the ovule. arrowhead, ovule primordium; *, nucellus; o, ovule; p, placenta. Scale bars are $40 \mu \mathrm{m}$ 

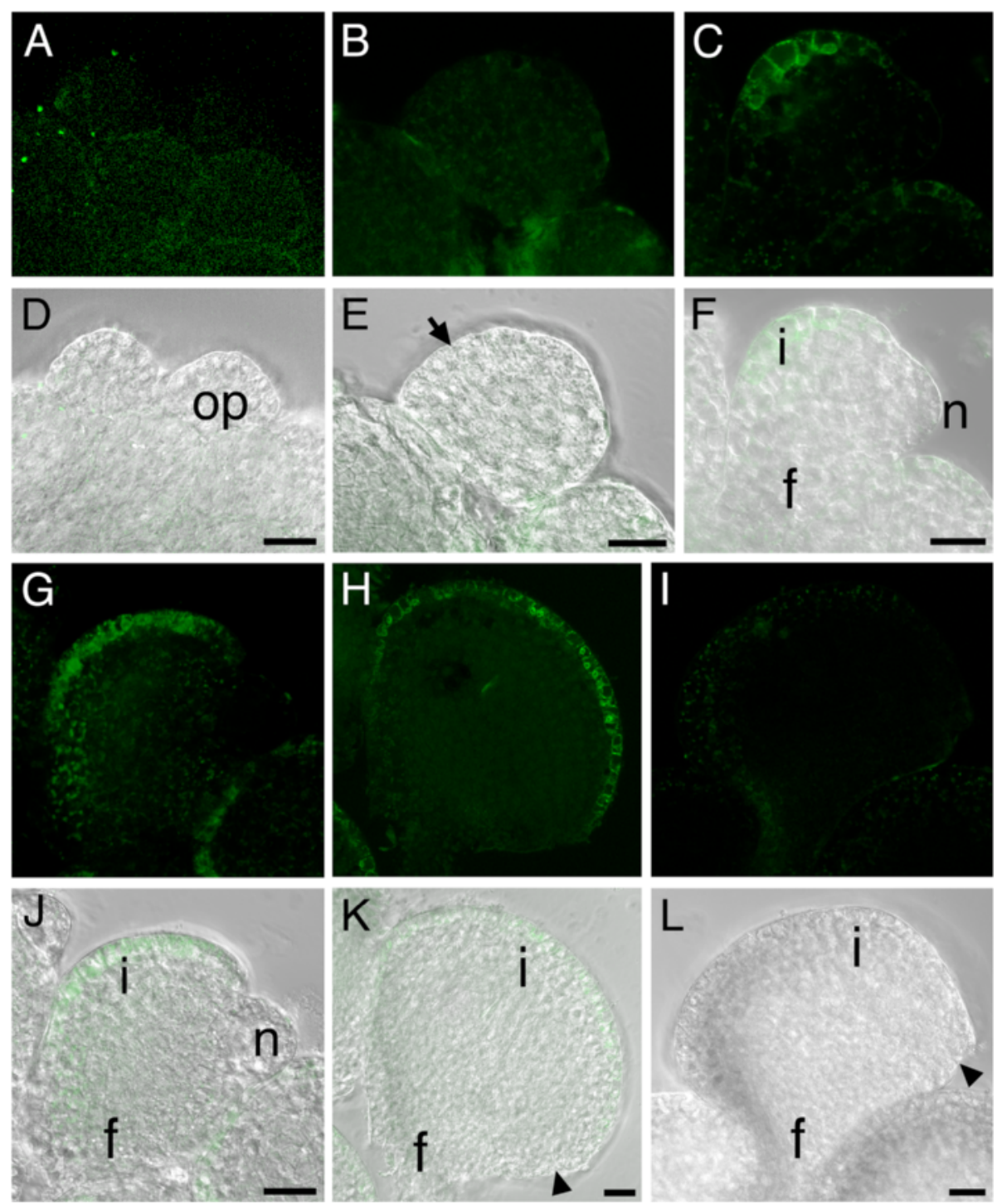

Fig. 2 Timing and specificity of a putative SIINO promoter directing expression of an SIINO-GFP transgene. Confocal images (a-c, $\mathbf{g - i}$ ) overlaid on corresponding DIC images $(\mathbf{d}-\mathbf{f}, \mathbf{k}-\mathbf{I})$ of tomato ovules. Control ovule with no transgene is shown in $\mathbf{i}, \mathbf{I}$. In this ovule the visible fluorescent dots are likely to be auto-fluorescence from plastids. All other images are from plants containing a P-SIINO::SIINO-GFP transgene. No GFP signal is present in stage 8 ovule primordia prior to integument initiation $(\mathbf{a}, \mathbf{d})$ or at stage 10, when asymmetric thickening of the primordium (arrow) signals emergence of the integument (b, e). GFP localized in the outermost cell layer is visible in the newly emerged integument primordium in stage 11 (c, f). This pattern continues as the integument grows asymmetrically to cover the nucellus in stage $13(\mathbf{g}$, j) and form the micropyle in the mature ovule at stage $18(\mathbf{h}, \mathbf{k})$. Expression is low or absent in the cells at the distal tip of the integument $(\mathbf{c}, \mathbf{g}, \mathbf{h})$.op, ovule primordium; f, funiculus; i, integument; n, nucellus; o, ovule; p, placenta; arrowhead, micropyle. Scale bars are $20 \mu \mathrm{m}$

$\mathrm{k}$ to Fig. 3c, d, i) and VIGS is well established in this species. A genomic fragment containing most of the coding region of NbINO1 (1162 bp) was used in a Tobacco Rattle Virus (TRV) silencing system [21]. Both NbINO1 and NbINO2 mRNA can be targeted for suppression by a single construct based on their high level of sequence identity (cDNAs are $97 \%$ identical, Additional file 5: Figure S4a), and because the genomic clone used includes regions of perfect identity of 64, 65, 73, 85 and 148 bp between the two genes (Additional file 5: Figure S4b), and only 23 to
$33 \mathrm{bp}$ of identity are required for effective VIGS [32, 33]. From three separate experiments, a total of eleven plants treated with the silencing construct and eight control plants treated with the empty vector were compared 4-8 weeks after co-infiltration with Agrobacteria containing the constructs and the pTRV1 helper vector. Two to five carpels from each plant were examined with SEM and/or light microscopy (Fig. 3). The silencing system was shown to be functional by control infiltrations of a VIGS plasmid designed to silence phytoene desaturase and in all three 

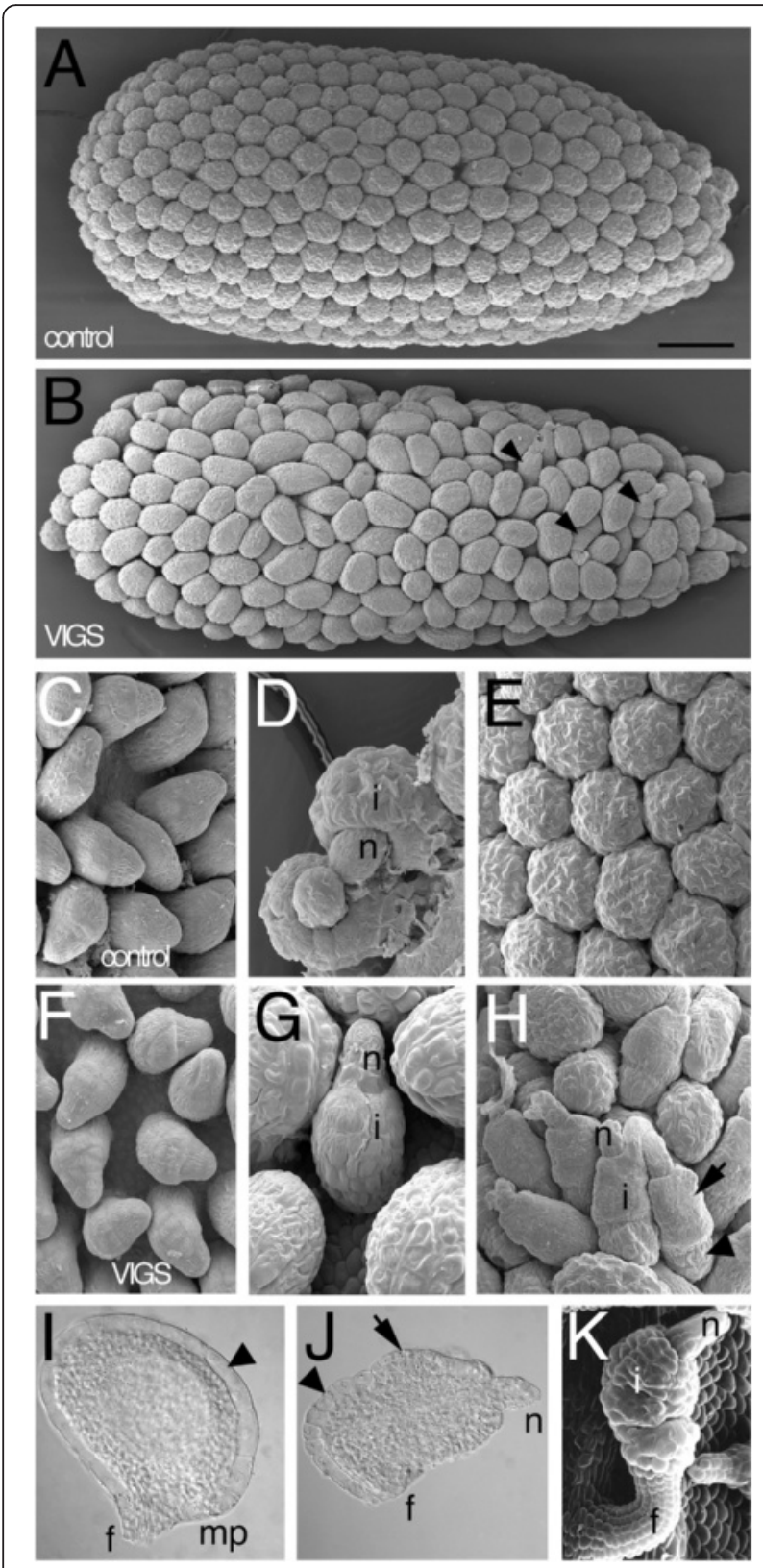

Fig. 3 VIGS knock-down of NbINO activity in N. benthamiana ovules. a Ovary of control carpel shows round and symmetrical ovules packed tightly on the placenta. $\mathbf{b}$ In a representative knock-down carpel the ovules are variable in shape and some are incompletely formed (examples indicated with arrowheads). Ovules shown in $\mathbf{c}-\mathbf{e}, \mathbf{i}$ are from control plants and $\mathbf{f}-\mathbf{h}, \mathbf{j}$ are knock-down ovules at similar stages. In $\mathbf{c}$ and $\mathbf{f}$ the integument appears as a thickening of the chalazal area and causes bending of the ovule primordium. The integument grows asymmetrically (d) while in knock-down ovules (g) growth of the integument is reduced. At maturity (e) the nucellus is no longer visible and ovules appear isodiametric. Knock-down ovules (h) are variable in shape and some are aberrant, with the nucellus protruding from a variable integument. The large cells of the outer cell layer of the integument (arrowhead) visible in $\mathbf{i}$ are only covering the basal part of the integument in $\mathbf{j}$. Smaller dense cells form the outermost layer of the distal part of the integument (arrow). $\mathbf{k}$ Arabidopsis ats ino double mutant ovule resembles the VIGS knock-down tobacco ovules. i: integument; f: funiculus; n: nucellus; mp: micropyle. Scale bar in a represents $250 \mu \mathrm{m}$ in $\mathbf{a}, \mathbf{b} ; 50 \mu \mathrm{m}$ in $\mathbf{c}, \mathbf{f} ; 40 \mu \mathrm{m}$ in $\mathbf{d}, \mathbf{g}, \mathbf{k} ; 125 \mu \mathrm{m}$ in $\mathbf{e}$; $100 \mu \mathrm{m}$ in $\mathbf{h} ; 80 \mu \mathrm{m}$ in $\mathbf{i}, \mathbf{j}$

experiments all such plants $(n=2,2$ and 1$)$ produced the expected white leaves [21].

Development of the tobacco ovule is essentially identical to that described for tomato. In early stages of development, in wild type plants, the integument was first visible as a thickening on one side of the chalazal region of an ovule primordium (Fig. 3c). When the leading edge of the growing integument became clearly defined the ovule bent and the tip of the nucellus was directed toward the placental wall (Fig. 3d). During this time the ovules can be at various stages of development within a carpel. After further growth of the integument, the nucellus was fully enclosed, the ovules increase in diameter and mature wildtype ovules at anthesis appeared as tightly packed rounded structures (Fig. 3a, e). At this stage, the micropyle was not visible as it was tucked underneath the ovule due to asymmetric growth of the integument (Fig. 3a, e, i).

In VIGS knock-down plants, early growth of the integument appeared similar to that in control plants (Fig. 3c and f), but subsequently some ovules showed reduced curvature (Fig. 3g). In knock-down plants at anthesis, most ovules (50-100\% of ovules from nine carpels examined using SEM) differed from wild type, appearing elongated or oval in shape (Fig. 3b), not symmetrically round as in untreated plants (Fig. 3a). Severely aberrant ovules (Fig. 3b, h) with exposed nucelli resulting from a shortened integument were observed from every treated plant, with numbers ranging from 1 to 27 severely aberrant ovules in a single carpel (35 carpels examined from eleven plants, Additional file 6: Table S2). The total number of ovules in one carpel ranged from 100 to 140 . Up to $20 \%$ of ovules were severely aberrant, depending on the plant and experiment (Fig. 3b, h). The variable effects on ovules were likely 
due to variability in the efficiency of the VIGS in different plants, experiments, and locations on the placenta, but such ovules were never observed in non-silenced control plants treated with the empty vector $(17$ carpels from 8 plants). These severely affected ovules were not fully recurved and were sometimes nearly linear, and the nucellus was exposed due to incomplete development of the integument (Fig. 3h, j). The incomplete integument comprised two regions, a rounded basal region that had large cells in the outermost layer of the integument (Fig. 3h, j), similar to the cell layer covering the entire control ovules (Fig. 3i), and a thinner distal region with smaller evenly shaped cells. The ovules with the most aberrant overall morphology were those with the most reduced region of large surface cells (Fig. 3j).

The most severely affected ovules closely resemble ovules of the Arabidopsis double mutant aberrant testa shape (ats) ino (Fig. 3k). In ats mutants the two integuments are congenitally fused into a single integument [34] more closely resembling solanaceous ovules than those of wild-type Arabidopsis. The ats ino double mutant shows the effect of Arabidopsis ino on a unitegmic ovule that derives from two fused integuments: reduced growth of the single integument, and growth comprising two clear regions (Fig. 3k) as seen in NbINO knockdown tobacco. Thus the failure in integument extension in the ovules of NbINO knock-down tobacco plants and the similarity between these ovules and those of the Arabidopsis double mutant indicate similar roles for INO in tobacco and Arabidopsis ovules.

\section{Interspecies transfer shows incomplete conservation of promoter and protein function}

INO gene expression and role in Solanaceae appears largely conserved with the INO gene in Arabidopsis and other studied plants. Such conservation of function may imply that the proteins and promoters have conserved specificities that can be tested by using heterologous transfer and observing the expression patterns and ability to complement the ino mutant.

A sequence comparison of either P-SlINO or a putative $\mathrm{P}-\mathrm{NbINO}(2 \mathrm{~kb}$ of sequence upstream of translation start site) with 5' sequence from Arabidopsis (P-AtINO) showed only very limited similarity that was confined to two regions previously defined as important for AtINO regulation, POS9 and POS6 [5, 35]. P-SlINO showed significant sequence conservation with $\mathrm{P}-\mathrm{NbINO}$ in only one region, around $-400 \mathrm{bp}$ to $-650 \mathrm{bp}$ using both LAGAN [36] and EARS [37] analyses (Additional file 7: Figure S5).

As a test of functional conservation of the promoters, $\mathrm{P}$-SIINO was used to drive expression of $ß$-glucuronidase (GUS) in Arabidopsis (Fig. 4). The ovule-specific expression of P-AtINO:GUS in Arabidopsis has been well characterized $[5,35]$, starting with visible expression at the

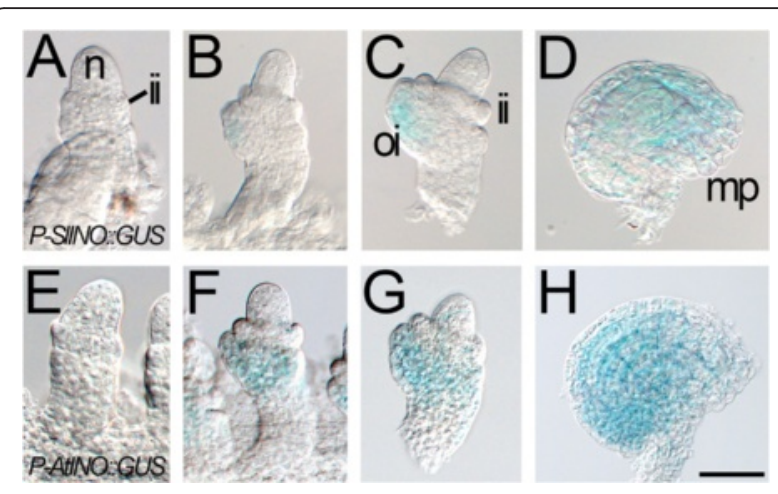

Fig. 4 Conservation of INO promoter shown by activity of P-SIINO in Arabidopsis (Ler), assayed using the GUS reporter. P-SIINO::GUS transgene activity ( $\mathbf{d}-\mathbf{g})$ compared with P-AtINO::GUS transgene activity in (e-h). Early ovules (a, e) show no GUS activity in the emerging outer integument. Expression of the reporter in the outer integument is visible in ovules at stage 2-IV [63] but P-SIINO expression (b) is less extensive than P-AtINO at the same stage (f). This trend continues as the integuments extend towards the nucellus at stage $3-1(\mathbf{c}, \mathbf{g})$. Finally, when the mature shape of the ovule is achieved at stage 4 -I the pattern of GUS activity in $\mathbf{d}$ and $\mathbf{h}$ are similar though expression directed by P-AtINO (h) is concentrated toward the basal end of the integument as previously noted [5] while expression directed by P-SIINO is concentrated more toward the distal end of the integument. o: ovule; p: placenta; mp: micropyle; c: carpel wall; $\mathrm{n}$ : nucellus; ii: inner integument; oi: outer integument. Scale bar in $\mathbf{h}$ represents $25 \mu \mathrm{m}$ in $\mathbf{a}-\mathbf{c}$, $\mathbf{e}-\mathbf{g}$; $50 \mu \mathrm{m}$ in $\mathbf{d}, \mathbf{h}$

site of outer integument initiation and maintenance in the outer integument during growth, though expression is concentrated at the base of the integument, rather than the growing tip (Fig. 4e-h). When P-SIINO:GUS was analyzed in Arabidopsis, expression was also ovule-specific and observed only in the outer integument (10 transformants analyzed). The P-SlINO and P-AtINO plants were stained simultaneously, and minor but consistent differences in pattern were observed. P-SIINO:GUS ovules showed lighter staining of the outer integument early in development (Fig. 4b, f) and, at maturity, staining was more concentrated at the distal tip, unlike the pattern observed for P-AtINO:GUS (compare Fig. 4d, h).

As a further test of functional conservation of the Arabidopsis and tomato promoter regions, P-SIINO was used to express the Arabidopsis AtINO coding region in an ino-1 mutant background to test for complementation of the mutant phenotype. Despite the expression pattern observed above, P-SlINO:AtINO was not able to complement the ino mutation, as outer integument growth was not restored in 6/6 ino-1 primary transformants (Fig. 5e). This result shows that even though there is a similar expression pattern from both promoters, there are critical aspects of the promoters that are not conserved.

To evaluate conservation of protein function, SIINO was produced in Arabidopsis under the control of PAtINO, to assay the protein's ability to complement the 


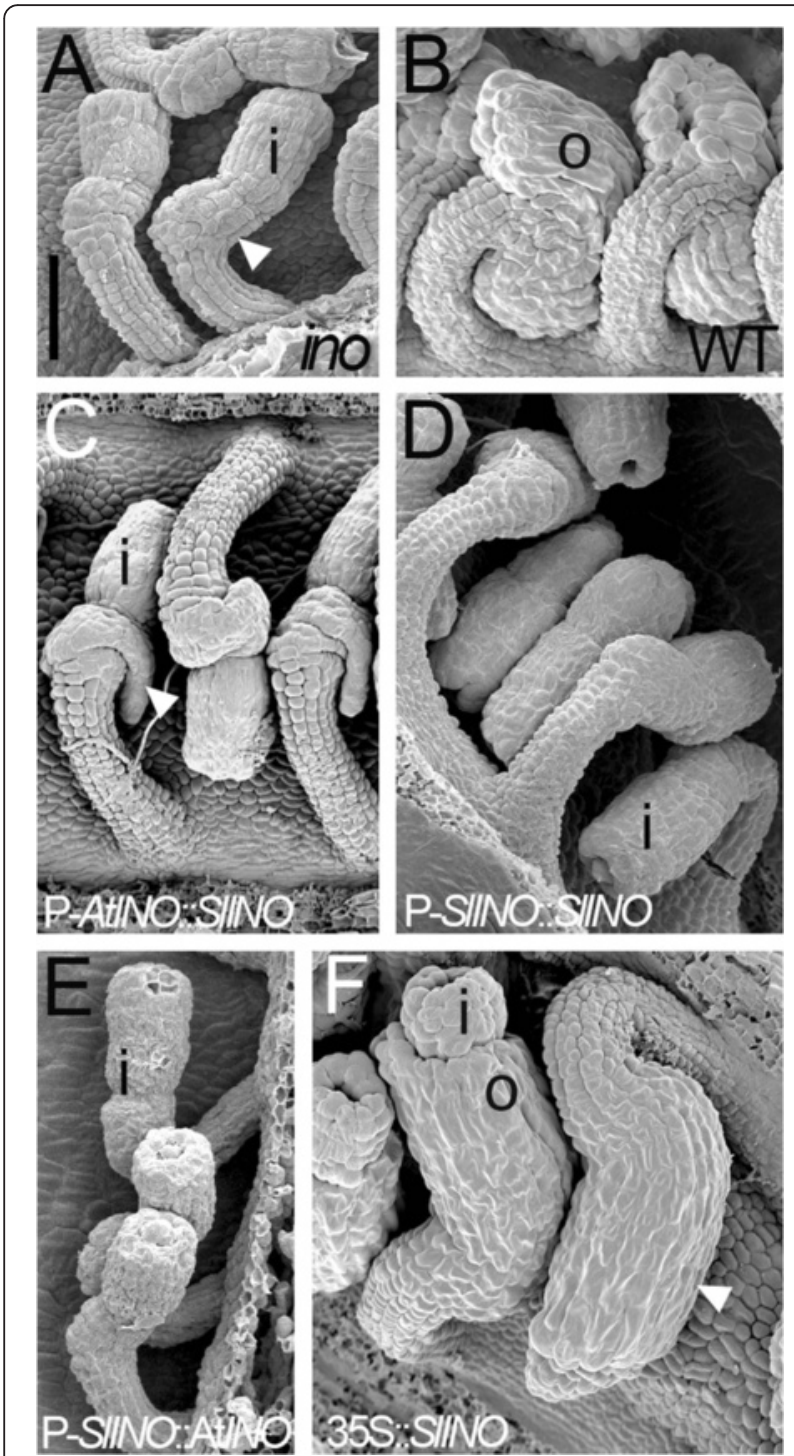

Fig. 5 Transgenic complementation experiments of Arabidopsis ino-1 by SIINO and AtINO expressed by P-AtINO, P-SIINO and the constitutive CaMV 355 promoter (all ovules at stage 4-I). ino-1 ovules (a) show the effect of loss of AtINO on outer integument growth. The inner integument is intact and where the outer integument should have grown to cover it, as in the wildtype (b), no integument was initiated on the gynobasal side (arrowhead). The SIINO protein, when expressed using P-AtINO (c), cannot complement ino-1 but leads to a small amount of misdirected growth (arrowhead) from the area where the outer integument should have arisen. This growth does not occur when P-SIINO is used to drive expression of SIINO (d) or AtINO (e). SIINO acts in a manner similar to other Arabidopsis YABBY genes when expressed using the constitutively active CaMV 355 promoter [64], promoting symmetrical growth of the outer integument (arrowhead) (f). This leads to a sup-like phenotype [5] in an ino-1 mutant background, showing that the SIINO protein is functional and can effect outer integument growth. i: inner integument; o: outer integument; WT: wildtype. Scale bar in a represents $50 \mu \mathrm{m}$ in panels a-e and $250 \mu \mathrm{m}$ in $\mathbf{f}$ ino mutant. In contrast to constructs with P-AtINO and the Arabidopsis AtINO coding region where a high frequency of full complementation was observed [35], the SIINO coding region was not effective at complementation. Four of six transgenic plants did not differ visibly from ino-1 mutants, while the remaining two plants showed minimal, aberrant outer integument growth (Fig. 5c). P-SlINO::SlINO was also not able to complement the ino- $1 \mathrm{mu}-$ tant (7/7 ino-1 primary transfomants Fig. 5d).

The failure of SIINO to complement ino-1 may indicate divergence in protein structure that leads to failure to act on downstream targets required for integument growth. As it has been shown that AtINO participates in auto-regulation leading to continued expression after initiation of expression [5], one such target may be PAtINO itself, with SIINO unable to maintain expression from P-AtINO. As a test of whether SIINO can function to support outer integument growth when expressed without regulatory constraints, a 35S::SlINO transgene was introduced into Arabidopsis (Ler) segregating for the ino-1 mutation. At least $25 \%$ of 42 transformants showed phenotypes commonly observed with ectopic overexpression of any active YABBY gene, such as curled leaves and carpels [38] (data not shown). The CaMV 35S promoter is not reliably expressed in ovules, but in $16 \%$ of the 42 independent transformants (all among those showing the vegetative phenotypic effects) there was evidence that SIINO was acting to promote outer integument growth. With the transgene in wildtype or heterozygous INO/ino backgrounds, symmetric growth of the outer integument was sometimes observed as compared with the normal asymmetric growth (3 plants). In four ino-1 transgenic plants, outer integument growth was variable but substantially more than usually observed in the mutant and more symmetrical than in wild-type plants (Fig. 5f). Taken together, these overexpression results indicate that SIINO is a functional YABBY protein that retains at least some ability to promote outer integument growth in Arabidopsis.

\section{Discussion}

The presence of two integuments is ancestral in the angiosperms [4] and the role of INO in integument development is conserved in examined bitegmic lineages in phylogenetically diverged clades $[6,7]$. The unitegmic ovules of solanaceous species represent an evolutionary innovation that is common to all euasterids [1]. The expression pattern of INO orthologs in the outer layer of integumentary cells has been shown to be conserved in all previously examined unitegmic species such as Prunus [28] and Impatiens [11], but a role for this gene in such ovules has not been revealed through loss or reduction of function until this study. 
The expression of INO in tomato is in only the outer integumentary layer preferentially on the side of the ovule with the greatest integument growth. This largely duplicates the pattern seen in all other angiosperms examined to date, including other members of the broader asterid clade (Impatiens) and members of the earliest branching angiosperm groups (Nymphaeales and Magnoliales). Hence it is reasonable to conclude that this pattern will also be in common with other members of the solanales with similar ovules such as tobacco. Indeed the effects of knock-down of NbINO in tobacco directly affect this layer, leading to a dramatic alteration in integument growth. The most affected ovules in the knock-down plants do not resemble those of loss of INO function in Arabidopsis or Annona (the only plants where such mutants have been examined), where the outer integument is completely absent and the inner integument grows normally and covers the nucellus. Instead the tobacco ovules appear to have reduced growth of the outermost portion of the single integument with continued proliferation of the interior material as an amorphous structure (Fig. 3h,j). While not resembling the Arabidopsis ino single mutant, the severely affected tobacco ovules are very similar in appearance to ovules of the Arabidopsis ino ats double mutant (Fig. 3k) [34]. The ats mutation causes congenital integument fusion, and the resulting unitegmic ovules in Arabidopsis resemble those of normal solanaceous plants [34]. The aberrant integument of both the tobacco knock-down and ino ats mutant ovules exhibit two surface cell types with small distal cells and larger cells on the surface of the proximal region. The origin of the distal region in the ino ats mutant is the inner layers of the single integument [34], and in tobacco the distal region derives from inner integumentary cell layers now exposed by reduction of the outer layer of enlarged cells. The extent of integument growth and resulting lack of ovule curvature in the knock-down plants correlated with the extent of the enlarged cells on the surface of the integuments. These cells correspond to those in which SIINO is expressed in tomato (and all other examined angiosperms), and the variability in growth of this region likely relates to variability in the effectiveness of gene suppression. Thus, in both tobacco and in Arabidopsis ats ino mutants, the loss or reduction in INO function leads to reduced outer cell layer proliferation and the resulting reduced and aberrant integumentary structures. The similarity of the effects of ino mutations on these two classes of unitegmic ovules indicates that the developmental role of INO is conserved between Arabidopsis and Solanales. Further, the most parsimonious explanation of the striking similarity of ovules produced in these two cases is that the single integument in Solanales evolved by congenital fusion of the ancestral inner and outer integuments (as produced by the ats mutant in Arabidopsis). Notably, one feature of Impatiens ovules that helped to identify unitegmic ovules as fused structures in that genus was retention of the differentiated endothelium as the inner layer of the single integument that corresponded to an anatomically similar layer in the inner integuments of bitegmic species [11]. The inner layer of ovules of the Solanaceae, such as tomato, also differentiates into an obvious endothelium [39]. Having an outer layer expressing the SIINO gene, and an inner layer differentiating into an endothelium, therefore, further supports the contention that the solanaceous integument is a fusion of ancestral outer and inner integuments.

Integument fusion was the cause of unitegmy in Impatiens [11] and, in a unitegmic Prunus species, reduced ATS and lack of production of ETTIN, an interaction partner of ATS, have been suggested as causing the evolution of unitegmy by fusion [28]. A similar mechanism may have been involved in the derivation of unitegmic ovules in the euasterids. Examination of this hypothesis could be the subject of future work.

Combining the results of species examined to date, we suggest that the role of INO in most species is to promote growth of the outer cell layer of a single or outer integument. This growth supports proliferation and organization of other attached layers to form an appropriately shaped laminar structure. Asymmetric distribution of INO expression results in asymmetric growth and the recurved, anatropous form of the ovules. In Arabidopsis, the spatial pattern of AtINO expression is controlled by SUPERMAN (SUP), which confines expression of AtINO to one side of the ovule by interfering with autoregulation [5]. The activity of SUP in the ovule appears to be confined to regulation of AtINO as ino mutations are fully epistatic to sup in the ovule [5]. This pathway appears to be conserved in the unitegmic ovules of Petunia hybrida, another Solanaceous species, where a mutation in SUP produces a phenotype similar to the Arabidopsis sup mutant - relatively symmetrical growth of the integument on all sides of the ovule [40]. This implies that the pathway controlled by SUP is conserved in the Solanaceae. Since SUP acts through regulation of $I N O$, this provides further evidence for conservation of the role of INO in promotion of integument growth in the Solanaceae.

A conserved role is often expected to be associated with a conserved protein function. However, our results indicate that despite clear orthology and conserved developmental roles, tomato and Arabidopsis INO proteins are not interchangeable as SIINO could not complement an Arabidopsis ino mutant when expressed from P-AtINO. This is in contrast to similar experiments with other paralogous Arabidopsis YABBY proteins, which promote varying levels of outer integument growth [38, 41] despite lower levels of amino acid identity with AtINO (30 \% to $35 \%$ ) 
than SIINO shares with AtINO (43\%). This result suggests that there are specific residues required for proper function of AtINO which are missing in SIINO, consistent with prior data that showed important determinants of AtINO function are distributed throughout the protein, rather than in a single structural domain [38, 41]. Taken together our results show that failure to complement in a heterologous expression experiment is not a reliable indicator that the proteins have diverged in their biological roles in the plants from which they derive.

In Arabidopsis, a functional AtINO protein is required for continued expression of P-AtINO, indicating autoregulation [5]. Thus, it is possible that the failure of SIINO to complement is due to either failure to activate P-AtINO, or failure to affect other downstream targets. We find that, when expressed under the CaMV 35 S promoter, SIINO can promote integument growth indicating that it is capable of properly affecting downstream targets. Thus it could be that the major failure of SIINO in Arabidopsis is an inability to autoregulate through $\mathrm{P}$-AtINO. However, it is also possible that SIINO has a reduced activity and overexpression from the $35 \mathrm{~S}$ promoter can overcome this and produce sufficient activity to produce the necessary downstream effects. The current results do not allow differentiation of these two hypotheses.

Developmental regulators, including $I N O$, are commonly expressed in spatially and temporally restricted zones, often mediated by strict transcriptional regulation. The domain of expression of INO in Arabidopsis and tomato is very similar with slightly different timing relative to initiation of the integument. The putative promoter of SIINO shares very little sequence identity with P-AtINO which is not unexpected due to the relative lack of conservation of promoter sequences. Remarkably, P-SlINO is able to produce in Arabidopsis a pattern of expression that is largely the same as that of the endogenous promoter suggesting that the native regulatory factors must be able to act on the non-native promoters despite their apparent lack of visible similarity. The slight differences observed in Arabidopsis - somewhat later initiation of expression, and expression tending more toward the distal end of the integument - echo the pattern of expression of the endogenous gene in tomato. This indicates that some of the differences in expression between these species derive from differences in the promoter sequences rather than only from differential presence of regulatory factors. Despite the similar expression pattern, P-SIINO::AtINO was not able to complement the ino-1 mutation, perhaps due to late initiation of expression, or due to failure to autoregulate as has been shown to be necessary for P-AtINO [5]. Alternatively, while the 5'-region of the Arabidopsis INO gene has been shown to be a fully functional promoter [35] it is possible that the tomato promoter relies on distal elements that are missing from the tomato genomic fragment used.

Of all the YABBY family members INO seems most conserved in function across angiosperms. In all species studied, except those containing very recent duplications (e. g. Nicotiana benthamiana), there is only a single INO gene, and loss of function phenotypes of Annona, tobacco and Arabidopsis point to a conserved role. In contrast, there is evidence that the role of other YABBY genes may have diverged significantly. In general, the FIL, YAB2 and $Y A B 5$ sub-families are expressed and function in laminar leaf growth while the $C R C$ and $I N O$ families play roles in reproductive structures, carpels and ovules, respectively $[8$, 42]. However, orthologs of $C R C$ are expressed and function in leaf vasculature (DROOPING LEAF in rice, [43]), and nectary development (CRC, [44]). Gains of function in these cases may be related to changes in both expression and protein function. Similarly, an ortholog of $Y A B 2$, the FASCIATED gene of tomato, is an important determinant of fruit shape, affecting carpel number [22, 45] and is expressed in carpels, placenta and stamens as well as ovules. The apparently stronger evolutionary constraint on INO's role may result from its importance in shaping integument architecture, which is crucial for efficient pollination and formation of the seed coat.

\section{Conclusions}

Our results show that INO orthologs in the Solanaceae are essential to the growth and expansion of the outer layer(s) of ovule integuments, and hence the ancestral role of $I N O$ is largely conserved in the derived unitegmic ovules of this group. The results further indicate that the unitegmic ovules of tobacco (and hence other euasterid species) are most likely the result of a congenital fusion of two ancestral integuments. Thus, integument fusion produced unitegmy in the euasterids as was found for Impatiens in the closely related Ericales. This may indicate that the common ancestor to these groups was already genetically predisposed to this evolutionary pathway. The tomato INO ortholog was ineffective at complementing the Arabidopsis ino mutant, despite being more conserved in sequence than are other paralogous YABBY gene sequences that were effective at complementation. This indicates that specific residues along the protein are critical to species-specific function. In addition, it shows that cross-species complementation is not always an effective means to evaluate the conservation of the functional role of orthologous genes in different species.

\section{Methods}

Plant material

Solanum lycopersicum varieties Micro Tom (for in situ hybridizations) and Moneymaker (all transgenic work) 
and Nicotiana benthamiana were obtained from the Ralph M. Parsons Plant Transformation facility at U. C. Davis. S. lycopersicum variety VF36 was obtained from the Tomato Genetics Resource Center at U. C. Davis. Arabidopsis (Landsberg erecta) ino-1 mutant was previously published [6].

\section{DNA constructs}

A tomato INO (SIINO) cDNA clone was isolated from a tomato pistil cDNA library constructed in $\lambda$-GT10 from strain VF36 [25] using the Arabidopsis AtINO cDNA [6] as a hybridization probe. The sequence of this clone differs by a single base from the cDNA predicted from gene Solyc05g005240.1.1 in the reference tomato genome [20]. The base substitution produces a conservative substitution of a valine in the VF36 cDNA for a corresponding isoleucine in the reference sequence. The tomato cDNA was used to screen a tomato genomic library [26] to isolate a genomic clone that included the 2257 bp of 5' flanking sequence extending up to the next putative gene (Solyc05g005250.2.1, pantothenate kinase). The clone also included four SIINO exons that confirmed that the single base change observed in the cDNA was a natural polymorphism between VF36 and the reference genome.

The SIINO cDNA coding region was inserted between the promoter and 3' flanking regions of AtINO as previously described [35] to create a P-AtINO::SlINO::AtINO3' transgene (pRKK170). The 2257 promoter region of SIINO (P-SIINO) was combined with the SlINO cDNA as a translational fusion with the GREEN FLUORESCENT PROTEIN (pGFP1.1.5, [46]) and the nopaline synthase $3^{\prime}$-end (Nos3') to make P-SIINO::SlINO:GFP::NOS3' (pRB30). P-SlINO was inserted into pHK17 [47] to drive expression of $E$. coli ß-glucuronidase (GUS, [48]) in a P-SIINO::GUS::NOS3' transgene (pRB8). P-SIINO was combined with both the AtINO cDNA and Nos3' to make P-SINO::AtINO::NOS3' (pRB67(71)) and with SIINO cDNA and Nos3' to make P-SIINO::SIINO::NOS3' (pRB66(68)) which were used to assess complementation in Arabidopsis.

\section{Plant transformation}

For plant transformation, chimeric transgenes were transferred as NotI fragments into pART27 [49] or pMLBART [49], which confer kanamycin or phosphinothricine resistance on transformed tomato or Arabidopsis plants, respectively. Transformations of the tomato Moneymaker variety were performed with Agrobacterium LBA4404 [50] at the Ralph M. Parsons plant transformation facility by published methods [51]. Transformation of Arabidopsis (Landsberg erecta) utilized either Agrobacterium LBA4404 or ASE [52] and the floral dip method [53].

\section{Virus induced gene silencing}

Genomic NbINO was amplified using Phusion Hifi taq polymerase with primers F1 atctagaATGTCAGCATT GAATCATCTGTTTG and R1 actcgagCTTTGGCATC TTTCTGTCTCC adding Xba1 and Xho1 sites. The product was blunt cloned into pJET1.2 (ThermoFisher, Waltham, MA) according to manufacturer's directions to make pDS207, which was sequenced to verify insert integrity. The insert was transferred using Xba1/Xho1 digestion and ligation into pYL156, the TRV2 vector $[54,55]$, to make pDS208. pDS208 was transferred into Agrobacterium GV2260 and used for VIGS via infiltration of $N$. benthamiana leaves [21] in three separate experiments. A control VIGS experiment using PHYTOENE DESATURASE (NbPDS) showed white leaves by 2 weeks after infiltration. Ovules from NbINO silenced plants were examined with SEM from 4 weeks after infiltration and compared to plants infiltrated with an empty silencing vector.

\section{Microscopy}

GUS staining followed the methods of Meister et al. [5]. Stained and unstained ovules were visualized under differential interference contrast (DIC) using a Zeiss Axioplan 2 microscope. Confocal images of tomato ovules expressing SIINO-GFP fusion protein were taken with a Zeiss LSM 710 system using $488 \mathrm{~nm}$ laser excitation and recording 493-598 nm emission wavelengths (Carl Zeiss Inc, Germany). For scanning electron micrography (SEM), Arabidopsis tissue was fixed and critical point dried as previously described [11], and tobacco carpels were treated similarly but each fixation step was twice as long, and three additional five minute soaks in liquid $\mathrm{CO}_{2}$ were used during critical point drying. Samples were imaged on a Philips XL 30 SEM (FEI Company, http://www.fei.com). In situ hybridizations on tomato (Microtom) followed the procedures described in Skinner and Gasser [56]. The hybridization probe derived from a 531 bp fragment isolated by PCR from the SIINO cDNA and containing the $3^{\prime}$ untranslated region and regions encoding the YABBY and central diverged domains. This fragment was inserted into pCRII-TOPO (Invitrogen/ThermoFisher, Carlsbad, CA) oriented with the $5^{\prime}$-end adjacent to the Xhol site. Digestion with XhoI and transcription with SP6 RNA polymerase (Promega, Madison, WI) produced the antisense hybridization probe.

\section{Additional files}

Additional file 1: Figure S1. Phylogenetic relationships of YABBY proteins from representative species. Amino acid sequences of YABBY proteins from representative species (all known YABBY proteins from Arabidopsis thaliana (At), Cabomba caroliniana (Cc), tomato (SI), rice (Os), 
and Amborella trichopoda (Amb), and example sequences from Antirrhinum majus (Am), Vitus vinerfa (Vv), Nymphaea alba and colorata ( $\mathrm{Na}$ and $\mathrm{Nc}$ ) and Pinus stichensis (Ps, used as the root) were aligned using Clustal X v. 2.1 [57]. Phylogenetic relationships were evaluated for maximum parsimony in PAUP 4.0b10 [58] using a heuristic search and the branch and bound algorithm. Characters were weighted using a BLOSUM62 [59] matrix as described [11]. The illustrated phylogram is the single shortest tree which was found in more than $49 \%$ of 1000 replicate searches. Branch lengths are proportional to number of changes in arbitrary units. Statistical significance of clades was evaluated with 1000 bootstrap resamplings using the same search criteria but with five replicate searches for each resampling. Bootstrap values $\geq 50 \%$ are shown on the supported clades. Sources of tomato and tobacco sequences are in Additional file 2: Table S1 and Additional file 3: Figure S2, and Genbank accession numbers of the other sequences are listed at the end of each taxon. Labels at far right are the five cannonical clades of YABBY proteins, color coordinated with the associated clade. The CRC and INO clades have especially strong statistical support. (PDF 356 kb)

Additional file 2: Table S1. Proposed S. lycopersicon tomato orthologs of Arabidopsis YABBY gene family members ([22], Fig. S1). (DOCX $52 \mathrm{~kb}$ )

Additional file 3: Figure S2. CLUSTAL O (1.2.1) multiple sequence alignment of Arabidopsis INO, and orthologous proteins from S. Iycopersicon (Solyc05g005240, SIINO) and N. benthamiana (our predicted version of Niben101Scf09599g00012.1, NbINO1 and Niben101Scf04287g04009.1, NblNO2). (DOCX $114 \mathrm{~kb})$

Additional file 4: Figure S3. The P-SIINO:SIINO-GFP transgene continues to be expressed after fertilization during the onset of fruit development. A-C: Ovules from P-SIINO:SIINO-GFP plants. D, E: Ovules from control plants. Images $\mathbf{A}$ (confocal) and $\mathbf{B}$ (DIC overlaid with GFP channel) show expression in the outer cell layer in an ovule postanthesis. C-E are images of the surface cells of the integument of ovules taken from 3-4 $\mathrm{mm}$ fruits. $\mathbf{C}$ and $\mathbf{D}$ are images taken on an epifluorescence microscope (Axioplan) using a Chroma GFP filter set 41017 (Chroma, Bellows Falls, VT). E is a dark-field image of the same ovule in $\mathbf{D}$. These images show expression is present in developing fruit. Scale bar in $\mathbf{B}$ represents $20 \mu \mathrm{m}$, scale bar in $\mathbf{E}$ represents $20 \mu \mathrm{m}$ in C-E. (TIF $4435 \mathrm{~kb}$ )

Additional file 5: Figure S4. Alignment of the two N. Benthamiana INO sequences showing regions of identity. A shows alignment of the coding sequences of Nb/NO1 and Nb/NO2 and $\mathbf{B}$ shows alignment of genomic fragment used for VIGS. Underlined regions are long stretches of identical sequences likely to be effective for VIGS and blue regions indicate exons. Alignment was performed using an implementation of the NeedlemanWunsch algorithm [60] as implemented at http://www.ebi.ac.uk/Tools/ psa/emboss_needle/nucleotide.html. (DOCX $107 \mathrm{~kb}$ )

Additional file 6: Table S2. Results of VIGS experiments showing the number of plants and carpels analyzed. (DOCX $64 \mathrm{~kb}$ )

Additional file 7: Figure S5. Comparison of INO promoters. A, B, and C show results of EARS analysis [37]. X-axis represents $P$-values for maximum alignment score for a $60 \mathrm{bp}$ window from each promoter to its best matching window in the Arabidopsis sequence $(\mathbf{A}, \mathbf{B})$ or tobacco $(\mathbf{C})$. A P-value less than $10^{-4}$ indicates conservation. D shows the result of a multispecies comparison of promoters using the LAGAN method [36] and VISTA tools [61] (http://genome.lbl.gov/vista/mvista/submit.shtml). Promoters from tobacco, tomato and Brassica oleracea (P-Bo/NO) are compared with P-AtINO (y-axis). Pink regions visible in P-BolNO comparison indicate high conservation of the POS6, POS9 and Minimal Promoter regions [62] of which only a small part of POS9 and POS6 are conserved at a very low level in the promoters from tobacco and tomato. (PDF $340 \mathrm{~kb}$ )

\section{Abbreviations}

ATS, Abberant testa shape; CaMV, cauliflower mosaic virus; CDNA, complementary DNA; CRC, CRABS CLAWS; DIC, differential interference contrast; FIL, filamentous flower; GUS, $\beta$-glucuronidase; GFP, green fluorescent protein; INO, inner no outer; SUP, superman; TRV, tobacco rattle virus; VIGS, virus induced gene silencing; YAB2/3/5, YABBY 2/3/5.

\section{Acknowledgements}

We thank Savithramma Dinesh-Kumar for providing materials, Stephen Gross for technical assistance, and members of the Gasser lab for helpful discussions.

\section{Funding}

Supported by U. S. National Science Foundation Grants IBN9983354 and IOS1354014 to CSG.

\section{Availability of data and materials}

All supporting data can be found within the manuscript and its additional files.

\section{Authors' contributions}

RKK isolated the tomato INO CDNA, performed in situ hybridizations and constructed a complementation vector; RHB isolated tomato genomic clones and made and evaluated expression and complementation constructs; DJS performed additional analysis of expression, complementation experiments, isolation of tobacco clones, and VIGS analysis. CSG supervised all work. DJS and CSG wrote the manuscript. All authors read and approved the final manuscript.

\section{Competing interest}

The authors declare that they have no competing interests.

\section{Consent to publish}

Not applicable.

Ethics approval and consent to participate

Not applicable.

\section{Author details}

'Department of Molecular and Cellular Biology, University of California, Davis, Davis, CA 95616, USA. ${ }^{2}$ Present address: US Patent and Trademark Office, 400 Dulany St, Alexandria VA 22314, USA. ${ }^{3}$ Present address: Department of Biological Sciences, University of Wisconsin-Whitewater, Whitewater, WI 53190, USA

Received: 7 December 2015 Accepted: 20 June 2016

Published online: 27 June 2016

\section{References}

1. Endress PK. Origins of Flower Morphology. In: Wagner GP, editor. The Character Concept in Evolutionary Biology. San Diego: Academic; 2001. p. 493-510.

2. Doyle JA, Endress PK. Morphological phylogenetic analysis of basal angiosperms: comparison and combination with molecular data. Int J Plant Sci. 2000;161(S6):S121-53.

3. Endress PK, Igersheim A. Gynoecium structure and evolution in basal angiosperms. Int J Plant Sci. 2000;161 (6 Suppl):S211-23.

4. Endress PK. Angiosperm ovules: diversity, development, evolution. Ann Bot-London. 2011;107(9):1465-89.

5. Meister RJ, Kotow LM, Gasser CS. SUPERMAN attenuates positive INNER NO OUTER autoregulation to maintain polar development of Arabidopsis ovule outer integuments. Development. 2002;129(18):4281-9.

6. Villanueva JM, Broadhvest J, Hauser BA, Meister RJ, Schneitz K, Gasser CS. INNER NO OUTER regulates abaxial-adaxial patterning in Arabidopsis ovules. Genes Dev. 1999:13(23):3160-9.

7. Lora J, Hormaza II, Herrero M, Gasser CS. Seedless fruits and the disruption of a conserved genetic pathway in angiosperm ovule development. Proc Natl Acad Sci U S A. 2011;108(13):5461-5.

8. Yamada T, Yokota S, Hirayama Y, Imaichi R, Kato M, Gasser CS. Ancestral expression patterns and evolutionary diversification of YABBY genes in angiosperms. Plant J. 2011;67(1):26-36.

9. Bouman F, Calis JIM. Integumentary shifting- A third way to unitegmy Ber Dtsch Bot Ges. 1977:90:15-28.

10. Albach DC, Soltis PS, Soltis DE. Patterns of embryological and biochemical evolution in the Asterids. Syst Bot. 2001;26(2):243-62.

11. McAbee JM, Kuzoff RK, Gasser CS. Mechanisms of derived unitegmy among Impatiens species. Plant Cell. 2005;17(6):1674-84.

12. Albach DC, Soltis PS, Soltis DE, Olmstead RG. Phylogenetic analysis of asterids based on sequences of four genes. Ann Mo Bot Gard. 2001;88(2):163-212.

13. Martin GW. Development of the flower and embryo-sac iu Aster and Solidago (concluded). Botanical Gasette. 1892;17(12):406-11. 
14. Vincent CA, Coen ES. A temporal and morphological framework for flower development in Antirrhinum majus. Can J Bot. 2004;82(5):681-90.

15. Brukhin V, Hernould M, Gonzales N, Chevalier C, Mouras A. Flower development schedule in tomato Lycopersicon esculentum cv. sweet cherry. Sex Plant Reprod. 2003;15(6):311-20.

16. Cooper DC. Macrosporogenesis and the development of the macrogametophyte of Lycopersicon esculentum. Am J Bot. 1931;18:739-48.

17. De Martinis D, Mariani C. Silencing gene expression of the ethylene-forming enzyme results in a reversible inhibition of ovule development in transgenic tobacco plants. Plant Cell. 1999;11(6):1061-72.

18. Angenent GC, Franken J, Busscher M, Van Dijken A, Van Went $J$, Dons HJM, Van Tunen AJ. A novel class of MADS box genes is involved in ovule development in petunia. Plant Cell. 1995;7(10):1569-82.

19. Colombo L, Franken J, Koetje E, Van Went J, Dons HJM, Angenent GC, Van Tunen AJ. The petunia MADS box gene FBP11 determines ovule identity. Plant Cell. 1995;7(11):1859-68.

20. Consortium TG. The tomato genome sequence provides insights into fleshy fruit evolution. Nature. 2012;485(7400):635-41.

21. Hayward A, Padmanabhan M, Dinesh-Kumar SP. Virus-induced gene silencing in Nicotiana benthamiana and other plant species. Methods Mol Biol. 2011;678:55-63.

22. Huang Z, Van Houten J, Gonzalez G, Xiao H, van der Knaap E. Genome-wide identification, phylogeny and expression analysis of SUN, OFP and YABBY gene family in tomato. Mol Genet Genomics. 2013;288(3-4):111-29.

23. Han HQ, Liu Y, Jiang MM, Ge HY, Chen HY. Identification and expression analysis of YABBY family genes associated with fruit shape in tomato (Solanum lycopersicum L.). Genet Mol Res. 2015;14(2):7079-91.

24. Bartholmes C, Hidalgo O, Gleissberg S. Evolution of the YABBY gene family with emphasis on the basal eudicot Eschscholzia californica (Papaveraceae). Plant Biol (Stuttg). 2012;14(1):11-23.

25. Gasser CS, Budelier KA, Smith AG, Shah DM, Fraley RT. Isolation of tissue-specific cDNAs from tomato pistils. Plant Cell. 1989;1:15-24.

26. Budelier KA, Smith AG, Gasser CS. Regulation of a stylar transmitting tissue-specific gene in wild-type and transgenic tomato and tobacco. Mol Gen Genet. 1990;224:183-92.

27. Bombarely A, Rosli HG, Vrebalov J, Moffett P, Mueller LA, Martin GB. A draft genome sequence of Nicotiana benthamiana to enhance molecular plantmicrobe biology research. Mol Plant Microbe Interact. 2012;25(12):1523-30.

28. Lora J, Hormaza Jl, Herrero M. Transition from two to one integument in Prunus species: expression pattern of INNER NO OUTER (INO), ABERRANT TESTA SHAPE (ATS) and ETTIN (ETT). New Phytol. 2015;208(2):584-95.

29. Xiao H, Radovich C, Welty N, Hsu J, Li D, Meulia T, van der Knaap E. Integration of tomato reproductive developmental landmarks and expression profiles, and the effect of SUN on fruit shape. BMC Plant Biol. 2009;9:49.

30. Burch-Smith TM, Anderson JC, Martin GB, Dinesh-Kumar SP. Applications and advantages of virus-induced gene silencing for gene function studies in plants. Plant J. 2004:39(5):734-46.

31. Liu Y, Nakayama N, Schiff M, Litt A, Irish VF, Dinesh-Kumar SP. Virus induced gene silencing of a DEFICIENS ortholog in Nicotiana benthamiana. Plant Mol Biol. 2004;54(5):701-11

32. Lu R, Malcuit I, Moffett P, Ruiz MT, Peart J, Wu AJ, Rathjen JP, Bendahmane A, Day $L$, Baulcombe DC. High throughput virus-induced gene silencing implicates heat shock protein 90 in plant disease resistance. EMBO J. 2003;22(21):5690-9.

33. Thomas $C L$, Jones L, Baulcombe DC, Maule AJ. Size constraints for targeting posttranscriptional gene silencing and for RNA-directed methylation in Nicotiana benthamiana using a potato virus X vector. Plant J. 2001;25(4):417-25.

34. McAbee JM, Hill TA, Skinner DJ, Izhaki A, Hauser BA, Meister R, Venugopala Reddy G, Meyerowitz EM, Bowman UL, Gasser CS. ABERRANT TESTA SHAPE encodes a KANADI family member, linking polarity determination to separation and growth of Arabidopsis ovule integuments. Plant J. 2006;46(3):522-31.

35. Meister RJ, Williams LA, Monfared MM, Gallagher TL, Kraft EA, Nelson CG, Gasser CS. Definition and interactions of a positive regulatory element of the Arabidopsis INNER NO OUTER promoter region. Plant J. 2004;37(3):426-38.

36. Brudno M, Do CB, Cooper GM, Kim MF, Davydov E, Program NCS, Green ED, Sidow A, Batzoglou S. LAGAN and Multi-LAGAN: efficient tools for large-scale multiple alignment of genomic DNA. Genome Res. 2003;13(4):721-31.

37. Picot E, Krusche P, Tiskin A, Carre I, Ott S. Evolutionary analysis of regulatory sequences (EARS) in plants. Plant J. 2010;64(1):165-76.

38. Gallagher TL, Gasser CS. Independence and interaction of regions of the INNER NO OUTER protein in growth control during ovule development. Plant Physiol. 2008;147(1):306-15.
39. Gasser CS, Robinson-Beers K. Pistil Development. Plant Cell. 1993;5:1231-9.

40. Nakagawa H, Ferrario S, Angenent GC, Kobayashi A, Takatsuji H. The petunia ortholog of Arabidopsis SUPERMAN plays a distinct role in floral organ morphogenesis. Plant Cell. 2004;16(4):920-32.

41. Meister RJ, Oldenhof H, Bowman JL, Gasser CS. Multiple protein regions contribute to differential activities of YABBY proteins in reproductive development. Plant Physiol. 2005;137(2):651-62.

42. Sarojam R, Sappl PG, Goldshmidt A, Efroni I, Floyd SK, Eshed Y, Bowman JL. Differentiating Arabidopsis shoots from leaves by combined YABBY activities. Plant Cell. 2010;22(7):2113-30.

43. Yamaguchi T, Nagasawa N, Kawasaki S, Matsuoka M, Nagato Y, Hirano HY. The YABBY gene DROOPING LEAF regulates carpel specification and midrib development in Oryza sativa. Plant Cell. 2004;16(2):500-9.

44. Lee JY, Baum SF, Oh SH, Jiang CZ, Chen JC, Bowman JL. Recruitment of CRABS CLAW to promote nectary development within the eudicot clade. Development. 2005;132(22):5021-32.

45. Cong B, Barrero LS, Tanksley SD. Regulatory change in YABBY-like transcription factor led to evolution of extreme fruit size during tomato domestication. Nat Genet. 2008:40(6):800-4.

46. Schumacher K, Vafeados D, McCarthy M, Sze H, Wilkins T, Chory J. The Arabidopsis det3 mutant reveals a central role for the vacuolar $\mathrm{H}(+)$-ATPase in plant growth and development. Genes Dev. 1999;13(24):3259-70.

47. Harikrishna K, Jampates-Beale R, Milligan SB, Gasser CS. An endochitinase gene expressed at high levels in the transmitting tissue of tomatoes. Plant Mol Biol. 1996;30:899-911.

48. Jefferson RA, Kavanagh TA, Bevan MW. GUS fusions: B-glucuronidase as a sensitive and versitile gene fusion marker in higher plants. EMBO J. 1987;6:3901-7

49. Gleave AP. A versatile binary vector system with a T-DNA organisational structure conducive to efficient integration of cloned DNA into the plant genome. Plant Mol Biol. 1992;20(6):1203-7.

50. Hoekema A, Hirsch PR, Hooykaas PJJ, Schilperoort RA. A binary plant vector strategy based on separation of vir- and T-region of Agrobacterium tumefaciens Ti-plasmid. Nature. 1983;303:179-80.

51. Filatti JJ, Kiser J, Rose B, Comai L. Efficient transformation of tomato and the introduction and expression of a gene for herbicide tolerance. In: Nevins D, Jones R, editors. Tomato Biotechnology. New York, NY: Alan R. Liss; 1987. p. 199-210.

52. Fraley RT, Rogers SG, Horsch RB, Eichholtz DA, Flick JS, Fink CL, Hoffmann NL, Sanders PR. The SEV system: a new disarmed Ti plasmid vector for plant transformation. Bio/Technology. 1985;3(7):629-35.

53. Clough SJ, Bent AF. Floral dip: A simplified method for Agrobacteriummediated transformation of Arabidopsis thaliana. Plant J. 1998;16(6):735-43.

54. Liu Y, Schiff M, Dinesh-Kumar SP. Virus-induced gene silencing in tomato. Plant J. 2002;31(6):777-86.

55. Liu Y, Schiff M, Marathe R, Dinesh-Kumar SP. Tobacco Rar1, EDS1 and NPR1/NIM1 like genes are required for $\mathrm{N}$-mediated resistance to tobacco mosaic virus. Plant J. 2002;30(4):415-29.

56. Skinner DJ, Gasser CS. Expression-based discovery of candidate ovule development regulators through transcriptional profiling of ovule mutants. BMC Plant Biol. 2009:9:29.

57. Thompson JD, Gibson TJ, Plewniak F, Jeanmougin F, Higgins DG. The CLUSTAL*X windows interface: flexible strategies for multiple sequence alignment aided by quality analysis tools. Nucleic Acids Res. 1997;25(24):4876-82.

58. Swofford DL. PAUP*: phylogenetic analysis using parsominy (and other methods), version 4.0. Sunderland, Massachusetts: Sinauer Associates; 1999.

59. Henikoff S, Henikoff JG. Amino acid substitution matrices from protein blocks. Proc Natl Acad Sci U S A. 1992;89(22):10915-9.

60. Needleman SB, Wunsch CD. A general method applicable to the search for similarities in the amino acid sequence of proteins. J Mol Biol. 1970;48(3):443-53.

61. Frazer KA, Pachter L, Poliakov A, Rubin EM, Dubchak I. VISTA: computational tools for comparative genomics. Nucleic Acids Res. 2004;32(Web Server issue):W273-9.

62. Simon MK, Williams LA, Brady-Passerini K, Brown RH, Gasser CS. Positiveand negative-acting regulatory elements contribute to the tissue-specific expression of INNER NO OUTER, a YABBY-type transcription factor gene in Arabidopsis. BMC Plant Biol. 2012;12(1):214.

63. Schneitz K, Hulskamp M, Pruitt RE. Wild-type ovule development in Arabidopsis thaliana: a light microscope study of cleared whole-mount tissue. Plant J. 1995;7(5):731-49.

64. Kay R, Chan A, Daly M, McPherson J. Duplication of CaMV 355 promoter sequences creates a strong enhancer for plant genes. Science. 1987; 236(4806):1299-302 